Article

\title{
Superradiant MeV $\gamma$ Scattered by a Room-Temperature Spinor Quantum Fluid
}

\author{
Yao Cheng ${ }^{1, *,+}$, Ting-Han Lin ${ }^{2}$, Chih-Hao Lee ${ }^{2,3}$ and Shun-Chi Wu ${ }^{2,3, *}$ \\ 1 Department of Engineering Physics, Tsinghua University, Haidian, 100084 Beijing, China \\ 2 Department of Engineering and System Science, National Tsing Hua University, Hsinchu 30013, Taiwan; \\ s105013803@m105.nthu.edu.tw (T.-H.L.); chlee@mx.nthu.edu.tw (C.-H.L.) \\ 3 Institute of Nuclear Engineering and Science, National Tsing Hua University, Hsinchu 30013, Taiwan \\ * Correspondence: yao@tsinghua.edu.cn (Y.C.); shunchi.wu@mx.nthu.edu.tw (S.-C.W.); \\ Tel.: +886-922880120 (S.-C.W.) \\ $+\quad$ Tsinghua University Beijing abruptly breached the contract of leading Author YC in May 2017, which is \\ pending now.
}

Academic Editor: Helmut Cölfen

Received: 18 May 2017; Accepted: 28 June 2017; Published: 1 July 2017

\begin{abstract}
Recent reports have revealed the rich long-lived Mossbauer phenomenon of ${ }^{93 \mathrm{~m}} \mathrm{Nb}$, in which it has long been speculated that the delocalized ${ }^{93 \mathrm{~m}} \mathrm{Nb}$ undergoes Bose-Einstein condensation following an increase in the ${ }^{93 \mathrm{~m}} \mathrm{Nb}$ density beyond the threshold of $10^{12} \mathrm{~cm}^{-3}$ at room temperature. We now report on the superradiant Rayleigh of the M4 $\gamma$ at $662 \mathrm{keV}$ scattered into end-fire modes along the long axis of the sample, as evidence of Bose-Einstein condensation. We observed the Arago (Poisson's) spot in order to demonstrate a near-field $\gamma$-ray diffraction from a mm-sized $\gamma$ source, as well as a $\gamma$ interference beyond the Huygens-Fresnel principle. During the 107-day monitoring period, seven Sisyphus cycles of mode hopping appeared in the superradiance, which demonstrates the optomechanic bistabilty provided by the collective interaction between the spinor quantum fluid and the impinging $\gamma$ s. Condensate-light interaction produces a pm matter-wave grating to become a Fabry-Pérot resonator with a Q-factor on the order of $10^{20}$, from which end-fired $\gamma \mathrm{s}$ lase.
\end{abstract}

Keywords: long-lived Mossbauer effect; delocalized nuclear $\gamma$ excitation; superradiant Rayleigh scattering; photonic crystal; $\gamma$ laser; spinor quantum fluid; symmetry-protected phase; magnetoelectric effect; topological defects; Majorana fermions

\section{Introduction}

Bose-Einstein condensation (BEC) [1,2] is among the most spectacular of the quantum phenomena to have been discovered over the last century. Micro-particles condense into one state generating a unique macroscopic feature, as defined by the off-diagonal long-range order (ODLRO) [3,4]. The whole condensate gives a global response to an external drive, rather than being merely a superposition of random micro-activities. It is well known that eV-energy $\gamma$ can undergo BEC at the relatively high temperature of $4 \mathrm{~K}[5,6]$, when the massless $\gamma$ acquires a small mass in the quantum well to become an exciton-polariton. Room-temperature BEC occurs [7] when the Mossbauer $\mathrm{keV} \gamma$ of an eV mass exceeds a threshold density of $10^{12} \mathrm{~cm}^{-3}$. This work applies ORLRO scattering of impinging $\gamma$ rays to prove the room-temperature BEC of delocalized ${ }^{93 \mathrm{~m}} \mathrm{Nb} \gamma$ excitations.

Long-lived Mossbauer nuclides emit biphoton $\gamma \gamma$ or a biphoton pair to preserve the energy from releasing a phonon [7-11]. Once nuclear resonance occurs, a single nuclear $\gamma$ excitation delocalizes to billions of identical nuclei in the crystal. Delocalized $\gamma$ excitation acquires an $\mathrm{eV}$ mass and its spin orientation in the crystal, by absorbing the Nambu-Goldstone bosons of phonon and magnon [12]. The nuclear exciton spreads in a $\mu \mathrm{m}$ region over the photonic crystal of identical nuclei, and it carries no 
charge, but does carry a spin. However, the term "nuclear exciton" [13] fails to capture the important character of an antiferromagnetic nesting spin chains [9]. We, therefore, prefer the term "nuclear spin-density wave" (NSDW) [7]. The NSDW BEC of the M4 ${ }^{93 \mathrm{~m}} \mathrm{Nb} \gamma \gamma$ in this report is, thus, a spinor quantum fluid carrying four spin quanta and odd parity. Coherent $\gamma$ s scattered by the spinor quantum fluid constitutes a $\gamma$ laser, which is highly useful for biological nanoimaging.

Early NSDW investigations focused on the ${ }^{103 \mathrm{~m}} \mathrm{Rh}$ of one-hour half-life [7,9-11] activated by a 6-MeV bremsstrahlung source in Beijing. The NSDW subsequently entered three regimes (I, II, and III) as its density increased, which were demonstrated by the behaviors of Rabi splitting [7,14]. We summarized the phenomena observed in regimes I and II in the supplementary information of our previous report [9]. In this report we concentrate on regime III, as well as an additional new regime beyond it. Either increasing the ${ }^{103 \mathrm{~m}} \mathrm{Rh}$ density above a threshold of $10^{12} \mathrm{~cm}^{-3}$ or lowering the sample temperature using liquid nitrogen [8] caused a quantum phase transition into regime III, as characterized by the abrupt shrinkage of the Rabi splitting from $500 \mathrm{eV}$ back to the $50-\mathrm{eV}$ vacuum splitting. The Rabi splitting switched twice between two constant values every 20 minutes, revealing a "collapse and revival" [15] of the NSDW condensate in regime III. By cooling the sample already prepared in regime III to $78 \mathrm{~K}$ each time, the NSDW condensate of ${ }^{103 \mathrm{~m}} \mathrm{Rh}$ entered a new regime, in which the Rabi splitting of NSDW slowly varied between 50 and $500 \mathrm{eV}$ with frequencies in the $\mathrm{mHz}$ range [11].

The support in Beijing for activating the ${ }^{103 \mathrm{~m}} \mathrm{Rh}$ was terminated in 2008, at which point we moved the NSDW experiments to Hsinchu and prepared the ${ }^{93 \mathrm{~m}} \mathrm{Nb}$ with a 16 -year half-life using neutron activation [7]. The activated $\mathrm{Nb}$ single crystal was an oval plate $(13 \mathrm{~mm} \times 12 \mathrm{~mm} \times 1 \mathrm{~mm})$, which was applied to measure the magnetoelectric effect (ME) induced by the BEC. The $\gamma$ spectra of ${ }^{93 \mathrm{~m}} \mathrm{Nb}$ provided no biphoton information, until the $\gamma \gamma$ at the half-transition energy (15.4 keV) were recently released from another polycrystalline sample used in this report [9]. We continued to verify the ${ }^{93 \mathrm{~m}} \mathrm{Nb}$ NSDW BEC for many years, until a transverse ME symmetry depending on the sample geometry was seen $[7,11]$, recognized as the dynamics of low-lying bound states in the topological defects of NSDW quantum fluid. For example, Khaymovich and Silaev have discussed the nonlinear paramagnetic magnetization of vortices in the ${ }^{3} \mathrm{He}-\mathrm{B}$ superfluid, which is induced by the Zeeman interaction and exhibits many low-frequency resonances [16]. The smoking-gun resonances of Majorana fermions are caused by the interlevel spacing of $n \hbar \omega_{v} \sim n h 10^{5} \mathrm{~Hz}$ and the subharmonics of $\omega_{v} / n$, where $n$ is an integer. A comprehensive review of recent progress on Majorana fermions in topological superfluids is given by Mizushima et al. [17], which introduces many possible magnetization sources, e.g., the spontaneous ferromagnetic vortex core and the exotic pairing of bound states. We take the pronounced magnetoinductance and the nonlinear $\mathrm{ME}$ resonances below $\mathrm{kHz}$ frequencies observed in a previous report [7] to be a fingerprint of topological defects in the spinor quantum fluid. A BEC threshold density of $10^{12} \mathrm{~cm}^{-3}$ was then found, when the delocalization of ${ }^{93 \mathrm{~m}} \mathrm{Nb}$ and its corresponding ME signature spontaneously disappeared three years after the activation [7]. However, BEC and the ME were immediately recovered by reactivating the sample again using neutron irradiation. Monitoring the transition from BEC to a normal state is still ongoing. Interestingly, ${ }^{103 \mathrm{~m}} \mathrm{Rh}$ and ${ }^{93 \mathrm{~m}} \mathrm{Nb}$ have similar BEC threshold densities at room temperature.

The ${ }^{93 \mathrm{~m}} \mathrm{Nb}$ ME showed a memory of the applied field. The $20-\mathrm{Hz}$ transverse ME remained nearly symmetrical on the rotation of the sample at $1 \mathrm{~T}$ when the vertical field was increased from 0 to $1 \mathrm{~T}$, and this rotating symmetry was broken at $1 \mathrm{~T}$ when the field was decreased from $9 \mathrm{~T}$ to $1 \mathrm{~T}$ [11]. The fluxes trapped in the sample were demonstrated by distributing magnetic powders on the sample surface to reveal a spontaneous magnetization of the neutral vortices [11], which was similar to the magnetic vortex core in the ${ }^{3} \mathrm{He}-\mathrm{B}$ superfluid [17]. Furthermore, the ME response switched sign from an inductor to a negative inductor for an anticlockwise rotation of the sample through 360 degrees at $9 \mathrm{~T}$, and vice versa. This geometric phase acquired by rotating sample is a hallmark of the non-Abelian statistics corresponding to Majorana zero modes [17], which probably survive at the high field and at room temperature. These three observations together reveal a topological nontrivial 
state of the spinor quantum fluid [17]. A metal-insulator transition was revealed by the negative $\mathrm{mV}$ voltage crossing a residual resistance of $4 \mu \Omega$ at $4 \mathrm{~K}$ [7]. Magnetic fluxes in quantized vortices inhibit the electron transport inside the crystal; instead, electrons propagate at the edge states $[17,18]$. The activated sample became an anisotropic insulator. Further investigation of the ME response beyond previous reports $[7,11]$ reveals that the time reversal breaking quantum fluid is related to the symmetry-protected Weyl superfluid [17], which will be discussed in a future report.

\section{Results}

\subsection{Suppression of Atomic Channels}

We recently reported superradiant $\mathrm{Nb} \mathrm{K}$-lines along a long sample axis for an active sample having a geometry of $1 \mathrm{~mm} \times 1.5 \mathrm{~cm} \times 3 \mathrm{~cm}$ [9]. We draw an analogy with the superradiant Rayleigh scattered from cold and ultracold atoms $[19,20]$ and, in particular, the atomic condensate in a high-finesse cavity [21]. The impinging $\gamma$ assumes the role of a laser, while the sample assumes the role of a $\gamma$ cavity containing the NSDW condensate. There are three major mirrors in the cavity, namely the matter-wave grating induced by $\gamma \mathrm{s}$, the nuclei on the lattice sites, and the sample boundary. The boundary mirrors give rise to the end-fire modes, while the matter-wave mirror provides a high-finesse Fabry-Pérot resonator [22]. The additional mirror of the lattice gives rise to some important physics of biphotons, which are absent in the cold-atom case.

We also previously reported the collective nuclear scattering of two E2 $\gamma \mathrm{s}$ at $1173 \mathrm{keV}$ and at $1333 \mathrm{keV}$ emitted from a ${ }^{60} \mathrm{Co}$ source, as well as an M4 $\gamma$ at $662 \mathrm{keV}$ emitted from a ${ }^{137} \mathrm{Cs}$ source [9]. Neither the ${ }^{60} \mathrm{Co}$ source nor a ${ }^{152} \mathrm{Eu}$ source provided the end-fire $\gamma \mathrm{s}$ by lateral impinging (see Figure 1) due to the fact that their coherent lengths were shorter than the sample thickness of $1 \mathrm{~mm}$.

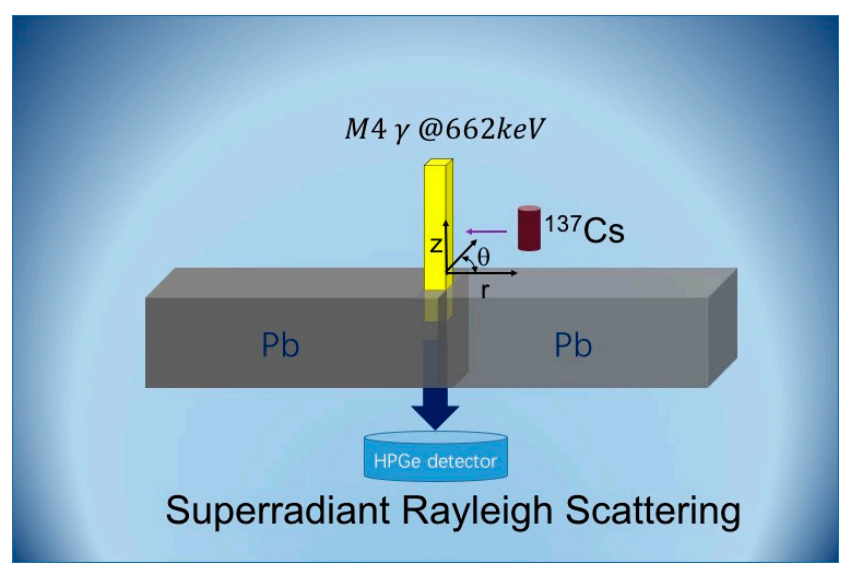

Figure 1. Schematic representation of the experiment. The activated sample $(1 \mathrm{~mm} \times 1.5 \mathrm{~cm} \times 3 \mathrm{~cm}$, yellow color) with the normal vector in the $\mathrm{E}-\mathrm{W}$ direction was surrounded by the $\mathrm{Pb}$ blocks $(8 \times 4 \mathrm{~cm} \times 8 \mathrm{~cm} \times 16 \mathrm{~cm})$. A $2.5-\mathrm{mCi}{ }^{137} \mathrm{Cs}$ point source was placed at the location of $(\mathrm{r}, \theta)$. The $\mathrm{Pb}$ blocks protected the HPGe detector from the leak exposure of ${ }^{137} \mathrm{Cs}$ source, except the end-fire mode (dark blue arrow) along the $\mathrm{z}$ axis. The lateral impinging multipolar $\mathrm{MeV} \gamma \mathrm{s}$ from ${ }^{137} \mathrm{Cs}$ gave rise to the superradiant Rayleigh scattering into the end-fire mode detected by a HPGe detector, which was located below the $\mathrm{Pb}$ shielding.

The resonant scattering of a Mossbauer nuclide takes place over $10^{-8} \mathrm{~s}$, which is much longer than the thermal vibration. The coherent elastic scattering channels are enhanced at Bragg, also known as the suppression of inelastic channels [13]. In our case, the condition of resonant Bragg scattering is no longer necessary because of the NSDW wave packet, in which the nuclei collectively enhance all kinds of elastic and inelastic nuclear scatterings. We, thus, use the term of SAC (suppression of atomic channels) rather than the "suppression of inelastic channels" in this report. 
$\mathrm{Nb}$ K-lines have a coherent length of $0.3 \mu \mathrm{m}$ [23] and a propagation depth of $50 \mu \mathrm{m}$ in the $\mathrm{Nb}$ crystal. The nucleus-field coupling $g_{\mathrm{K}}$ of $\mathrm{K}$ lines is no more than $10^{-8} \mathrm{eV}$, while the allowed E1 transitions of ${ }^{93} \mathrm{Nb}$ lie above $1.3 \mathrm{MeV}$. Nearly $10^{9}$ nuclei lie in the coherent length of $0.3 \mu \mathrm{m}$. The atom-field coupling is collectively enhanced by a factor of $\sqrt{N}$ for an ensemble of $N$ ultracold atoms [21]. In contrast, the ${ }^{93 \mathrm{~m}} \mathrm{Nb}$ NSDW entangles $N^{2}$ modes of $N$ nuclei $\times N$ phonons, giving rise to an $\mathrm{N}$-fold nucleus-field coupling [9]. However, $N \times g_{\mathrm{K}} \sim 10 \mathrm{eV}$ is still much weaker than large nuclear detuning. How can we determine the nature of the underlying physics to yield the superradiant $\mathrm{Nb}$ K-lines in a previous report [9], which shall be completely attenuated before reaching the sample boundaries? The penetration depth must be longer than $1 \mathrm{~mm}$. We cannot understand the $\mathrm{Nb} \mathrm{K}$-line superradiance without considering the NSDW condensate, which enhances the Borrmann penetration [13] of $\gamma s$ emitted from an internal source.

A ${ }^{152} \mathrm{Eu}$ source provides E1 and E2 $\gamma \mathrm{s}$ of coherent lengths longer than $\mu \mathrm{m}$. It also provides atomic E1 $\gamma$ s of the Sm K-lines, which have very short coherent lengths of $60 \mathrm{~nm}$ [23]. Table 1 shows a pronounced enhancement of the off-Bragg penetration at $122 \mathrm{keV}$ by $170 \sigma$. The E2 SAC showed up regardless of the large $622-\mathrm{keV}$ detuning. In contrast, the E1 SAC of Sm K-lines at $40 \mathrm{keV}$ and $45 \mathrm{keV}$ were absent. The nucleus-field coupling of the $\mathrm{E} 2 \gamma$ at $122 \mathrm{keV}$ is of the $\mu \mathrm{eV}$ order and its coherent length lies between the intrinsic $0.6 \mathrm{~m}$ and the thermal broadening of $60 \mu \mathrm{m}$. Accordingly, we identify the amplification as $N$ rather than $\sqrt{N}$, otherwise the collective coupling is too weak to give any observable SAC effect from an external ${ }^{152} \mathrm{Eu}$ source. Note that the NSDW wave packet has a size of $\mu \mathrm{m}$. There would be no SAC, unless ODLRO was present.

Table 1. Suppression of atomic channels (SAC) tested by a $30-\mu \mathrm{Ci}{ }^{152} \mathrm{Eu}$ source. All measurements were undertaken with a $2.5 \mathrm{~cm}$ detector-source spacing, where the active sample always remained at the same position. Calibration: source atop the spacer; Pristine: source atop the pristine sample; Active: source atop the active sample; DAS: relative deviation between active and pristine data in \%; DT: dead time of the HPGe detector. Assuming active and pristine count rates at $1408 \mathrm{keV}$ are equal, we multiply the active data by a factor of 1.0036 to give the relative deviations of DAS. Three datasets in cps (counts per second) were undertaken by daily exchanging the ${ }^{152} \mathrm{Eu}$ source positions to maintain the equal ${ }^{182} \mathrm{Ta}$ activities. $\gamma$ at $1121 \mathrm{keV}$ is emitted from the ${ }^{182} \mathrm{Ta}$ decay. The half-lives at $368 \mathrm{keV}$ and $779 \mathrm{keV}$ denoted by * are not documented, however, they are estimated from the E1 transition at $1408 \mathrm{keV}$.

\begin{tabular}{ccccccc}
\hline$\gamma$ in keV & $\gamma$ Type & Half-Life & Calibration & Pristine & Active & DAS \\
\hline 40 & E1 & $0.01 \mathrm{fs}$ & $15.6 \pm 0.1$ & 0 & 0 & - \\
45 & E1 & $0.01 \mathrm{fs}$ & $18.1 \pm 0.1$ & 0 & 0 & - \\
296 & E1 & $27 \mathrm{fs}$ & $5.78 \pm 0.05$ & $5.16 \pm 0.02$ & $5.16 \pm 0.01$ & $0.5 \pm 0.4$ \\
368 & E1 & $0.2 \mathrm{ps} *$ & $8.95 \pm 0.05$ & $7.35 \pm 0.02$ & $7.39 \pm 0.01$ & $1.0 \pm 0.3$ \\
444 & E1 & $27 \mathrm{fs}$ & $24.23 \pm 0.05$ & $22.29 \pm 0.02$ & $22.31 \pm 0.02$ & $0.5 \pm 0.1$ \\
779 & E1 & $0.2 \mathrm{ps} *$ & $60.86 \pm 0.07$ & $56.81 \pm 0.02$ & $56.73 \pm 0.02$ & $0.22 \pm 0.05$ \\
1408 & E1 & $27 \mathrm{fs}$ & $61.48 \pm 0.06$ & $59.05 \pm 0.02$ & $58.84 \pm 0.02$ & 0.0 \\
122 & E2 & $1.4 \mathrm{~ns}$ & $652.7 \pm 0.2$ & $376.18 \pm 0.05$ & $385.71 \pm 0.05$ & $2.90 \pm 0.017$ \\
245 & E2 & $58 \mathrm{ps}$ & $108.2 \pm 0.1$ & $94.02 \pm 0.03$ & $94.27 \pm 0.03$ & $0.62 \pm 0.04$ \\
344 & E2 & $32 \mathrm{ps}$ & $282.8 \pm 0.1$ & $252.04 \pm 0.04$ & $252.25 \pm 0.04$ & $0.44 \pm 0.02$ \\
964 & E2 + M1 & $0.87 \mathrm{ps}$ & $59.25 \pm 0.07$ & $56.46 \pm 0.02$ & $56.29 \pm 0.02$ & $0.05 \pm 0.05$ \\
1121 & E2 + M1 & $114.13 \mathrm{~d}$ & $133.54 \pm 0.09$ & $134.57 \pm 0.03$ & $134.39 \pm 0.03$ & $0.22 \pm 0.03$ \\
DT & - & - & $16.21 \%$ & $15.28 \%$ & $15.30 \%$ & - \\
\hline
\end{tabular}

The question remains of how to evaluate the $\mathrm{N}$-fold coupling to provide the E1 SAC. The E1 N-fold coupling of an $\gamma$ emitted from a two-level nuclide decreases very rapidly with its $\gamma$ energy at zero temperature while it remains a constant value when the thermal broadening dominates. Furthermore, the photoelectric attenuation decreases with the $\gamma$ energy. Regardless of which conditions apply, the E1 SAC is a monotonically decreasing function of the $\gamma$ energy. We shall accordingly identify the insignificant E1 SAC from their trivial attenuations. Three E1 $\gamma \mathrm{s}$ at $296 \mathrm{keV}$, at $368 \mathrm{keV}$, and at $444 \mathrm{keV}$ provide pronounced deviations in their coherent lengths caused by the multi-level transitions. The thermal broadening dominates the coherent length of $16 \mu \mathrm{m}$ at $368 \mathrm{keV}$, while the extreme short half-life of $27 \mathrm{fs}$ reduces the coherent lengths of $10 \mu \mathrm{m}$ at $296 \mathrm{keV}$ and $9 \mu \mathrm{m}$ at $444 \mathrm{keV}$, respectively. The $\mathrm{N}$-fold coupling at $368 \mathrm{keV}$ is stronger than that at $296 \mathrm{keV}$ and at $444 \mathrm{keV}$. Table 1 shows 
a superior E1 SAC at $368 \mathrm{keV}$ by $3 \sigma$. The SAC coherence beyond the NSDW size reveals the forward ODLRO superradiance.

\subsection{Superradiant Rayleigh Scattering and Its Near-Field Diffraction}

As shown in Figure 1, the $\mathrm{M} 4 \gamma$ at $662 \mathrm{keV}$ from a $2.5-\mathrm{mCi}{ }^{137} \mathrm{Cs}$ source irradiated the active sample from its lateral side, while detecting $\gamma \mathrm{s}$ along the 3 - $\mathrm{cm}$ long sample axis. The superradiant M4 $\gamma$ was verified with great care by two methods, in addition to the following Figures $2-5$, namely by replacing the active sample by a pristine one and blocking the irradiation path with a $2-\mathrm{cm}$-thick $\mathrm{Pb}$ brick. Figure 2 and Table 2 show detailed measurements of one and a half circles around the active sample. One mirror symmetry along the 1-mm axis was found, while the mirror symmetry along the $1.5-\mathrm{cm}$ axis was shown in rough terms.

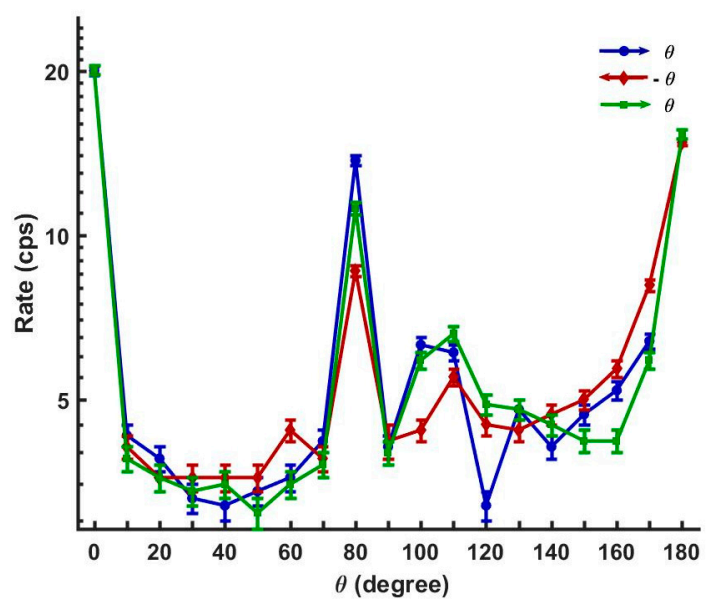

Figure 2. The $\theta$-dependence of the superradiant Rayleigh scatterings of $\mathrm{M} 4 \gamma$ at $662 \mathrm{keV}$ in counts per second (cps) as a function of the ${ }^{137} \mathrm{C}$ s source location of $(5.4 \mathrm{~cm}, \theta)$. Data listed in Table 2 were taken every five minutes for a sequence from 0 to 540 degrees $(-\theta$ means $360-\theta$ to demonstrate the mirror symmetry).

Table 2. $\theta$-dependence of superradiant Rayleigh in cps as a function of the source location of $(r, \theta)$, where $r$ is the radius and $\theta$ is the impinging angle, as shown in Figure $1.2 .5 \mathrm{mCi}{ }^{137} \mathrm{Cs} 3-\mathrm{mm}$ point source was placed on the circle $r=5.4 \mathrm{~cm}$ radius; the second column of configuration was measured in the upwards sequence of $-\theta$ from the bottom number to the top number, while the other two columns were measured in a downward sequence. The data were obtained every five minutes.

\begin{tabular}{cccc}
\hline$\theta$ in Degree & $\theta$ & $-\theta$ & $\theta$ \\
\hline 0 & $20.0 \pm 0.4$ & - & $20.1 \pm 0.4$ \\
10 & $4.3 \pm 0.2$ & $4.1 \pm 0.2$ & $3.9 \pm 0.2$ \\
20 & $3.9 \pm 0.2$ & $3.6 \pm 0.2$ & $3.6 \pm 0.2$ \\
30 & $3.3 \pm 0.2$ & $3.6 \pm 0.2$ & $3.4 \pm 0.2$ \\
40 & $3.2 \pm 0.2$ & $3.6 \pm 0.2$ & $3.5 \pm 0.2$ \\
50 & $3.4 \pm 0.2$ & $3.6 \pm 0.2$ & $3.1 \pm 0.2$ \\
60 & $3.6 \pm 0.2$ & $4.4 \pm 0.2$ & $3.5 \pm 0.2$ \\
70 & $4.2 \pm 0.2$ & $3.9 \pm 0.2$ & $3.8 \pm 0.2$ \\
80 & $13.7 \pm 0.3$ & $8.6 \pm 0.3$ & $11.2 \pm 0.3$ \\
90 & $4.1 \pm 0.2$ & $4.2 \pm 0.2$ & $4.0 \pm 0.2$ \\
100 & $6.3 \pm 0.2$ & $4.4 \pm 0.2$ & $5.9 \pm 0.2$ \\
110 & $6.1 \pm 0.2$ & $5.5 \pm 0.2$ & $6.6 \pm 0.2$ \\
120 & $3.2 \pm 0.2$ & $4.5 \pm 0.2$ & $4.9 \pm 0.2$ \\
130 & $4.8 \pm 0.2$ & $4.4 \pm 0.2$ & $4.8 \pm 0.2$ \\
140 & $4.1 \pm 0.2$ & $4.7 \pm 0.2$ & $4.5 \pm 0.2$ \\
150 & $4.7 \pm 0.2$ & $5.0 \pm 0.2$ & $4.2 \pm 0.2$ \\
160 & $5.2 \pm 0.2$ & $5.7 \pm 0.2$ & $4.2 \pm 0.2$ \\
170 & $6.4 \pm 0.2$ & $8.1 \pm 0.2$ & $5.9 \pm 0.2$ \\
180 & - & $14.8 \pm 0.3$ & $15.3 \pm 0.3$ \\
\hline
\end{tabular}


According to conventional wisdom, the pm-wavelength $\gamma$ from a mm source provides no information on the $\mathrm{cm}$ geometry because its fringe visibility [22] vanishes. To demonstrate this highly-unusual near-field diffraction, we studied the superradiance in detail for a few specific angles: $0,60,80$, and 90 degrees. The angular spread of impinging $\gamma$ was reduced by moving the source away from the sample step-by-step, as shown in Figure 3 and Table 3. The superradiance decreased faster than $1 / \mathrm{r}^{2}$, as shown by the results for $\theta=0^{\circ}$. The superradiant efficiency at $\left(1 \mathrm{~cm}, 0^{\circ}\right)$ approached $40 \%$, as estimated by the superradiance of $1.6 \times 10^{5}$ counts per second (cps) taking two end-fire modes, forward and backward superradiance in the 0 -degree direction, a $50 \%$ pile-up loss and a $20 \%$ efficiency of the directional beam (0.5\% efficiency of the coaxial HPGe detector at $662 \mathrm{keV}$ using an isotopically emitting source, as provided by the vendor) into account, while the impinging $\gamma \mathrm{s}$ are $4 \times 10^{5} \mathrm{~s}^{-1}$ in $0.5 \%$ of the $4 \pi$ solid angle without considering polarizations of the M4 $\gamma$. Meanwhile, the $40 \%$ superradiant efficiency also manifested the M4 SAC, otherwise the photoelectric loss of 662-keV $\gamma$ propagating $3 \mathrm{~cm}$ from the center to the end of sample would be more than $90 \%$.

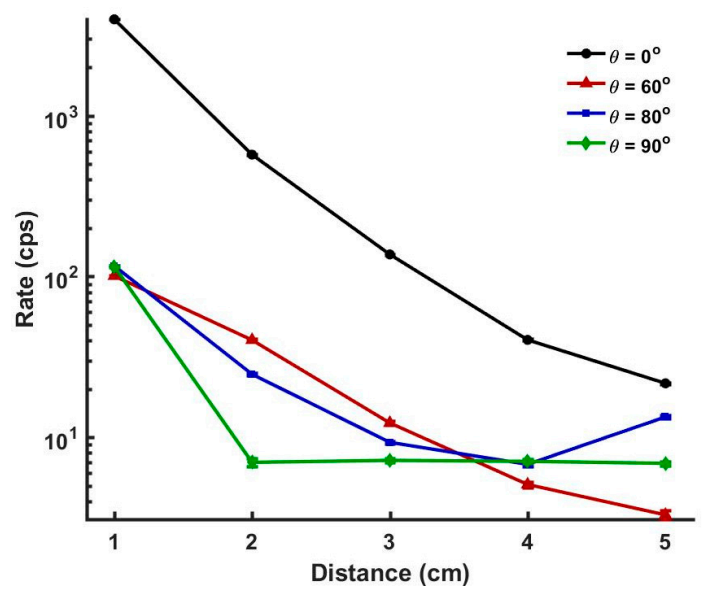

Figure 3. The r-dependence of superradiant Rayleigh scatterings of M4 $\gamma$ at $662 \mathrm{keV}$ in cps as a function of the ${ }^{137}$ Cs source location of $(r, \theta)$. Data listed in Table 3 were taken every five minutes.

Table 3. R-dependence of superradiant Rayleigh in cps as a function of source location of (r, $\theta)$. The source was $2.5 \mathrm{mCi}{ }^{137} \mathrm{Cs}$. A nodal point appeared at $\left(3.5 \mathrm{~cm}, 80^{\circ}\right)$, while the nearby low superradiances were at $\left(3.3 \mathrm{~cm}, 60^{\circ}\right)$ and $\left(3.7 \mathrm{~cm}, 90^{\circ}\right)$. Note that the normal value of the internal $\gamma$ at $1121 \mathrm{keV}$ was $6.92 \pm 0.01 \mathrm{cps}$. The readings at $\left(1 \mathrm{~cm}, 0^{\circ}\right)$ were lower than the real values, because of the pile-up loss of $48 \%$ dead time. The internal $\gamma$ at $1121 \mathrm{keV}$ was slightly increased by the ${ }^{137}$ Cs bombardment.

\begin{tabular}{cccccc}
\hline $\boldsymbol{\theta} / \mathbf{r}$ & $\mathbf{1} \mathbf{~ c m}$ & $\mathbf{2} \mathbf{~ c m}$ & $\mathbf{3} \mathbf{~ c m}$ & $\mathbf{4} \mathbf{~ c m}$ & $\mathbf{5} \mathbf{~ m ~}$ \\
\hline $0^{\circ} 662 \mathrm{keV}$ & $3973 \pm 5$ & $572.8 \pm 1.7$ & $137.3 \pm 0.6$ & $40.4 \pm 0.4$ & $21.7 \pm 0.3$ \\
$0^{\circ} 1121 \mathrm{keV}$ & $5.9 \pm 0.4$ & $6.9 \pm 0.2$ & $6.5 \pm 0.2$ & $7.1 \pm 0.2$ & $6.9 \pm 0.2$ \\
$60^{\circ} 662 \mathrm{keV}$ & $100.8 \pm 0.7$ & $40.3 \pm 0.5$ & $12.3 \pm 0.3$ & $5.1 \pm 0.2$ & $3.3 \pm 0.2$ \\
$60^{\circ} 1121 \mathrm{keV}$ & $6.8 \pm 0.2$ & $7.0 \pm 0.2$ & $7.2 \pm 0.2$ & $7.1 \pm 0.2$ & $7.2 \pm 0.2$ \\
$80^{\circ} 662 \mathrm{keV}$ & $116.5 \pm 0.8$ & $24.7 \pm 0.4$ & $9.3 \pm 0.2$ & $6.8 \pm 0.2$ & $13.4 \pm 0.3$ \\
$80^{\circ} 1121 \mathrm{keV}$ & $6.8 \pm 0.2$ & $6.7 \pm 0.2$ & $7.0 \pm 0.2$ & $6.9 \pm 0.2$ & $7.1 \pm 0.2$ \\
$90^{\circ} 662 \mathrm{keV}$ & $115.5 \pm 0.7$ & $22.3 \pm 0.4$ & $8.6 \pm 0.2$ & $5.3 \pm 0.2$ & $3.9 \pm 0.2$ \\
$90^{\circ} 1121 \mathrm{keV}$ & $7.0 \pm 0.2$ & $7.0 \pm 0.2$ & $7.2 \pm 0.2$ & $7.1 \pm 0.2$ & $6.9 \pm 0.2$ \\
\hline
\end{tabular}

Note that the superradiance in Table 3 was not a monotonic function at $\theta=80^{\circ}$. A nodal point was found at $\mathrm{r}=3.5 \mathrm{~cm}$. We also traced the nodal "low" that developed in the nearby positions. Vanishing superradiance was also found at the corresponding dark position $\left(1 \mathrm{~cm}, 60^{\circ}\right)$ using a large disk source of $10-\mu \mathrm{Ci}{ }^{137} \mathrm{Cs}$. Rotating this disk source changed the superradiance, i.e., a small angular distribution in the r-axis gave a large superradiance by an order of magnitude. Interference, thus, occurred 
among the incoherent $\gamma$ s of disjointed arrivals, otherwise summing over their single- $\gamma$ interference should provide a vanishing fringe visibility [22]. We, thus, conclude that NSDW condensate has a memory while the impinging $\gamma \mathrm{s}$ gain their mutual coherence in the resonator. Furthermore, the NSDW rotational symmetry was broken [9], as revealed by rotating the disk source.

About two hundred years ago in 1818, Siméon D. Poisson challenged the wave theory of light proposed by Augustin-Jean Fresnel that a bright spot appears in the shadow of an opaque disk [22]. Later, Dominique-François-Jean Arago provided the experimental proof of a bright spot behind a metallic disk, known as the spot of Arago (or Poisson's spot). We now demonstrate the near-field diffraction of $\mathrm{MeV} \gamma \mathrm{s}$ by a dark Arago spot. According to the Huygens-Fresnel principle the light is constituted as a propagating wave passing through homogenous space at an instantaneous moment, without considering past events. The non-Markovian superradiance of a $\mathrm{MeV} \gamma$-ray may be not only attributed to the matter-light interaction [21], but also to the non-Huygens nature of the n-dimensional wave equation of $n=1$ or $n=2$ [24].

\subsection{Room-Temperature BEC as Proven by End-Fire Superradiance}

The Rayleigh scattering demanded a recoil loss $<0.1 \mathrm{MeV}$, which allowed a cm-long interference among many $\gamma s$ laterally spread over wide impinging angles. Bounded nuclei at lattice sites take the recoil collectively, rather than the recoil energy $\omega_{R}=\hbar k^{2} / 2 m$ being taken by a single cold-atom of mass $\mathrm{m}$ [21]. The backaction of matter-wave grating guides the highly coherent superradiance along the long axis [21]. Ultracold atoms in the condensate move to specific positions, where the constructive interference redirects the impinging laser $\gamma \mathrm{s}$ into the end-fire modes, known as the self-organized Dicke phase $[21,25]$. The energy cost of moving entire $\mathrm{Nb}$ nuclei to match the impinging $\gamma$ phase is more than $10^{16} \mathrm{eV}$, as estimated by the Einstein phonon model with an average nuclear displacement of half pm. The theory of the Dicke transition reported in [21,25] is, thus, not applicable in our case. Instead, the standing-wave $\gamma$ s must be biphotons, which automatically match the bcc lattice. A soft mechanism fine-tunes the atomic positions or nuclear orientations to provide the matter-wave grating.

$\mathrm{M} 4 \gamma$ at $662 \mathrm{keV}$ mainly coupled with the $\mathrm{Nb} \mathrm{M} 4$ transition at $970 \mathrm{keV}$ to yield a nuclear detuning of $\Delta_{N}=308 \mathrm{keV}$. No extra $\mathrm{Nb}$ K-lines were found under an exposure of $4 \times 10^{7} \mathrm{M} 4 \gamma \mathrm{s}$ per second (see Figure S3A in the Supplementary Information). We set a SAC bound for the two-photon Rabi frequency, i.e., $\Omega_{c}^{2} / \Delta_{N} \gg 10 \mathrm{eV}[21,25]$. This implies $\Omega_{c}=N^{2} \times B \times g_{0} \gg 1 \mathrm{keV}$, where $g_{0} \sim 10^{-17} \mathrm{eV}$ is the light-matter coupling of single nucleus in free space, as estimated by the 2-min half-life of the M4 $\gamma$ emitted from ${ }^{137} \mathrm{Ba}, N$ is the number of nuclei and $\mathrm{B} \sim 10^{-11}$ is the factor of biphoton-pair branching [9]. Here, we apply a collective coupling of $N^{2}$ instead of $N$, because more than $10^{12}$ nuclei located in the coherent length of $\gamma$ is greater than 1/B. Collective coupling of $10^{15}$ nuclei provides rigorous evidence of the NSDW BEC. The strong coupling of $\Omega_{c} \sim \Delta_{N}$ traps the incident M4 $\gamma \mathrm{s}$, which dissipate at impurities [8,9]. Superradiant $\gamma \mathrm{s}$ of different energies coexist along the long sample axis, e.g., the E2 $\gamma$ at1121 keV emitted from the ${ }^{182} \mathrm{Ta}$ decay, as long as the coupling is fast enough to provide SAC.

How can the Å lattice provide the pm matter-wave grating and how can $\gamma$ s of different energies coexist? We provide an intuitive picture to interpret the underlying physics in this early stage of investigations. High-energy M4 $\gamma \mathrm{s}$ confine themselves due to the Kerr effect [21] caused by the axipetal density of the 1-D NSDW chains [9]. The $N^{2}$-rule implies that the matter-wave grating consists of phonons and magnons. Nuclei remain on their lattice sites but move away slightly by the collective phonon modes. The nuclear density incorporated with the nuclear orientation, thus, provide an average 1-D coupling varying at a pm scale.

\subsection{Mode Hopping of the Matter-Wave Grating}

We found a systematic oscillation of the superradiance in time. Figure 4 shows the first long-term monitoring experiment of the superradiant $\mathrm{M} 4 \gamma$ irradiated by the $2.5-\mathrm{mCi}{ }^{137} \mathrm{Cs}$ source located at $\left(0.3 \mathrm{~cm}, 90^{\circ}\right)$. Removing the smoothed results of Figure 4 from the raw data gives a variance of 
$25,630 \pm 90$, which is significantly beyond the variance of the shot noise, i.e., 24,000 , to reveal plenty of hidden signals in the fluctuation. Here, we concentrate on the quantized oscillations in this report, while the systematic analysis of signals of periods ranging from days to minutes will be discussed in greater detail elsewhere.

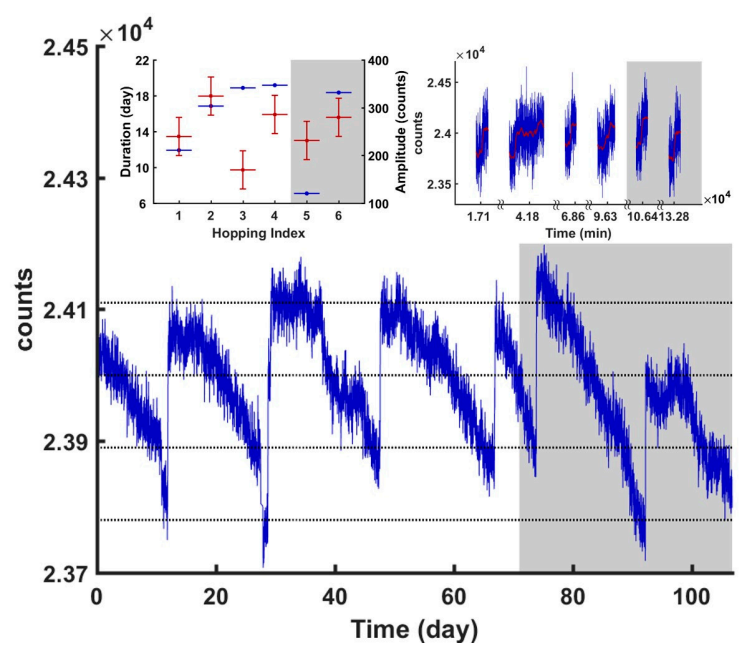

Figure 4. Seven Sisyphus cycles of superradiant Rayleigh scattering generated by a $2.5-\mathrm{mCi}{ }^{137} \mathrm{Cs}$ source at the position $\left(0.3 \mathrm{~cm}, 90^{\circ}\right)$ in the first long-term monitoring experiment begun on 1 August 2016 (day 0), the raw data of which are given in Figure S1 of the Supplementary information. The time sequence of data was $\gamma$ counts per minute, while the presentation incorporates hourly smoothing (61 data points). Four horizontal lines indicate the potential quantization. Air conditioners were turned off after day 71, indicated by the gray area. Inset diagrams show the mode hopping periods (blue), the hopping amplitudes (red) and the raw + smoothed data (blue + red) of the Sisyphus falls.

Assuming an unknown tick-tack clock $\Xi$ in the NSDW condensate drove seven Sisyphus cycles by increasing the effective susceptibility $\chi$, as the optomechanical bistability of the ultracold atoms driven by sweeping the laser frequency upwards [21,26]. Taking the upside-down Sisyphus "fall" to be a step function, the relaxation time is about half an hour. However, the M4 $\gamma$ at $662 \mathrm{keV}$ immediately extinguished and reestablished within one second when we removed and returned the ${ }^{137}$ Cs source, respectively.

Two air conditioners that maintained the temperature within $27 \pm 0.5^{\circ} \mathrm{C}$ were turned off at day 72. No extra fluctuation was observed by increasing the temperature fluctuation by an order of magnitude to $\pm 6^{\circ} \mathrm{C}$. The superradiant Rayleigh indeed consisted of pairing $\gamma \gamma$, e.g., $\gamma_{11} \gamma_{12}$ and $\gamma_{21} \gamma_{22}$ with energies $E_{11}+E_{12}+E_{21}+E_{22}=E_{0}(662 \mathrm{keV})$ and the wavelengths in vacuum $\sim 4 \lambda_{0}$. A slight shift of the standing-wave energy of $\mathrm{E}_{11}-\mathrm{E}_{12}=\mathrm{E}_{21}-\mathrm{E}_{22}=4.34 \mathrm{keV}+\delta$ [9] absorbs the $0.01 \%$ temperature elongation of the lattice constant $\mathrm{a}_{0}=2.86 \AA$ A between two nearest-neighbor $\mathrm{Nb}$ atoms. Free $\gamma_{11} \gamma_{12}+$ $\gamma_{21} \gamma_{22}$ combination to match different lattice constants together with entangled phonons and magnons provides the soft mechanism to launch the self-organized Dicke phase [21].

The mode hopping at $\sim 1 \%$ per Sisyphus cycle indicates a grating number of the matter-wave, i.e., $\mathrm{a}_{0} / \lambda_{0}$. Four possible quantizeFid levels are also highlighted in Figure 4 . If the tick-tack $\Xi$ is the collection of $0.01 \%$ impurities and the vacancy at lattice sties, missing or replacing ${ }^{93} \mathrm{Nb}$ all together gives a net effective $\chi$ on the new vacuum of NSDW condensate, i.e.:

$$
\chi=\sum_{i j} N_{j} \chi_{i ; j}(\boldsymbol{E} ; \boldsymbol{B})
$$

where $j$ runs for all kinds of impurities, $N_{j}$ is the number of impurities or vacancies, and $\chi_{i ; j}$ are their effective susceptibilities of the $i$ th order as functions of the $\boldsymbol{E}$ and the $\boldsymbol{B}$ fields. Every $\beta$ decay increases 
one nuclear charge at impurity sites and meanwhile increases their $\chi_{1 ; j}$ susceptibilities. Slight variation of $\chi_{1 ; j}$ repartitions the biphoton $\gamma \gamma$ energy like the temperature elongation, which hardly changes the $\gamma$ intensity. In contrast, the $\chi_{2}$ variation can be directly compensated by the blue-detuned $\gamma$ intensity, as demonstrated by the downward region in Figure 4. Hyper-Raman processes accelerated the ${ }^{94} \mathrm{Nb} \beta$ decay (see Figure S3C in the Supplementary information), which revealed a significant $\chi_{2}$ contribution.

Note that the M4 field of $10^{17} \mathrm{~V} / \mathrm{m}$ and $10^{5} \mathrm{~T}$ are available in cavities of impurity atoms, which may catalyze chemical reactions and nuclear reactions $[8,9]$. Evaporation of impinging $\gamma s$ at impurities, impurity decays, activation of ${ }^{93 \mathrm{~m}} \mathrm{Nb}$, and matter-wave back-action balanced each other during the downward region of the Sisyphus cycles until the mode hopping, which quickly advanced to the next resonance. Figure 5 shows the cross correlation among the ${ }^{182} \mathrm{Ta} \gamma \mathrm{s}$, and three broad-band $\gamma \mathrm{s}$ of BBL, BBM, and BBH, which represent three broad-band $\gamma \mathrm{s}$ below $662 \mathrm{keV}$, between 662 and $1121 \mathrm{keV}$, and above $1121 \mathrm{keV}$. The hopping of broad-band $\gamma$ s provides further evidence of matter-wave grating. Figure 5 also reveals two hidden hopping in Figure 4.

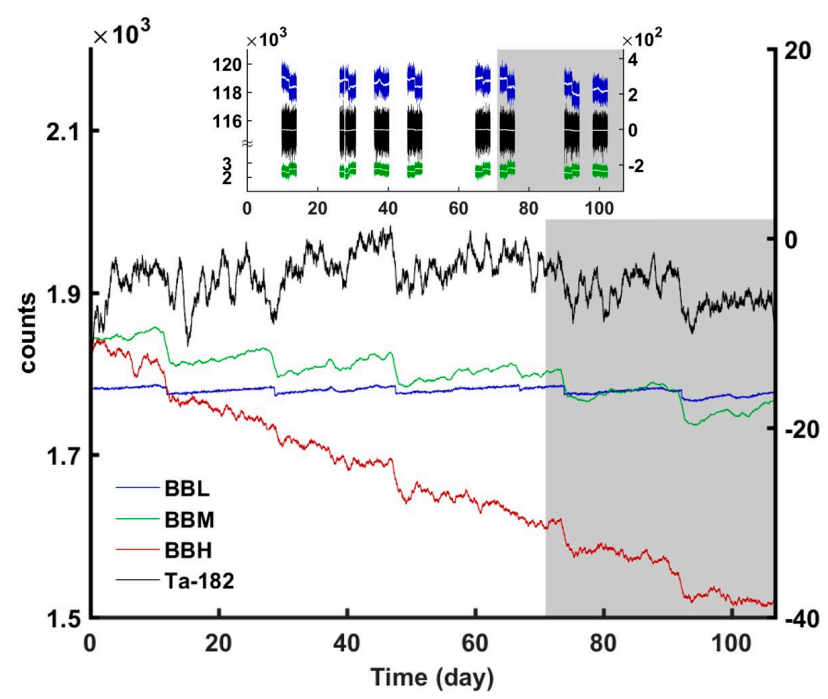

Figure 5. Fluctuations of ${ }^{182} \mathrm{Ta} \gamma \mathrm{s}$ and broad-band $\gamma \mathrm{s}$. The right-hand axis shows fluctuation of total counts of $\gamma \mathrm{s}$ at $1121,1189,1221$, and $1231 \mathrm{keV}$, which are collected to represent the ${ }^{182}$ Ta decay. Note that counts of ${ }^{182} \mathrm{Ta} \gamma \mathrm{s}$ were less than the calibrated baseline. The left-hand axis shows counts of $0.015 \times \mathrm{BBL}$, BBM, and $10 \times \mathrm{BBH}$, which represent three broad-band $\gamma$ s below $662 \mathrm{keV}$, between 662 and $1121 \mathrm{keV}$, and above $1121 \mathrm{keV}$. Fluctuations in these are presented against calibrated baselines, as detailed in the Supplementary Information. BBL incorporates hourly smoothing, while BBM, BBH, and ${ }^{182} \mathrm{Ta} \gamma \mathrm{s}$ incorporate daily smoothing. An inset diagram gives raw and smoothed (white) data of the Sisyphus falls. Air conditioners were turned off after day 71, indicated by the gray area.

Additional broad-band $\gamma \mathrm{s}$ (BBL, BBM, and BBH in Figure 5) were created at the moment of applying the M4 $\gamma$, as shown in Figures S2 and S3 of the Supplementary Information. For example, the BBL broad-band $\gamma \mathrm{s}$ of energy below the $662 \mathrm{keV}$ in Figure 5 was mainly contributed by the scattering of the $662-\mathrm{keV}$ biphoton fragments occurring at the sample site, rather than the Compton plateau occurring at the detector site. The BBL spectra in Figure S3A of the Supplementary Information show two bumps located near $630 \mathrm{keV}$ and $470 \mathrm{keV}$, which revealed a loss of $30 \mathrm{keV}$ by Raman pumping from ${ }^{93} \mathrm{Nb}$ to ${ }^{93 \mathrm{~m}} \mathrm{Nb}$ and broad distributions of the coincident arrival of four and three $\gamma \mathrm{s}$ of the biphoton fragments, respectively.

The highest $\gamma$ energy emitted from the ${ }^{182} \mathrm{Ta}$ decay is $1.453 \mathrm{MeV}$, which is limited by the $\mathrm{Q}_{\beta}$ of $1.814 \mathrm{MeV}$. However, we found decaying counts of a broad-band background which extended exponentially to an energy above $1.5 \mathrm{MeV}$, as shown in Figure S3C of the Supplementary Information, which strongly supports the reported $4-\gamma$ picture of standing-wave M4 $\gamma$ s trapped in the photonic 
crystal and released by NSDW annihilation. The coincident arrivals of high-energy $\gamma \mathrm{s}$ of the 662-keV biphoton fragments gives rise to broad-band counts distributed with an exponential shape, the $30-\mathrm{keV}$ low-energy version of which was previously reported [9].

\subsection{Surficial Superradiance}

Figure $\mathrm{S} 4$ in the Supplementary information shows a $0.4 \%{ }^{182} \mathrm{Ta}$ growth over two $\theta$-dependence measurements using two $10-\mu \mathrm{Ci}{ }^{137} \mathrm{Cs}$ sources from day-13 to day-2. Figures 5 and $\mathrm{S} 4$ both show a $2 \%$ systematic oscillation of the ${ }^{182} \mathrm{Ta} \gamma \mathrm{s}$ with periods of several days. Nearly $6 \times 10^{3}$ impinging $\gamma \mathrm{s}$ per second provided a $\theta$-dependent superradiance of the 0.01 -cps order, the single end-fire efficiency of which was $10^{-5}$. The superradiant efficiency increased 10 times using the $2.5-\mathrm{mCi}$ source at the same positions, as shown in Figure 3. It increased 10,000 times when the 2.5-mCi source was placed at the particular position of $\left(1 \mathrm{~cm}, 0^{\circ}\right)$. The dramatic increase of the superradiant efficiency reveals a non-uniform distribution of the superradiance. The matter-wave grating of M4 $\gamma$ suppressed the ${ }^{182} \mathrm{Ta}$ $\gamma \mathrm{s}$, as shown by the fast hopping response of the ${ }^{182} \mathrm{Ta} \gamma \mathrm{s}$ in Figure 5 . The fast reaction of ${ }^{182} \mathrm{Ta} \gamma \mathrm{s}$ on moving the source was also observed. However, the growth in Figure S4 remained after the source was removed. The growth might be an illusion of particular systematic oscillations lasting a month, as driven by an external source, e.g., the earth's magnetic field or the gravitational field.

We moved the ${ }^{137} \mathrm{Cs}$ source from the front side to the back side of the sample at $\left(0.3 \mathrm{~cm}, 270^{\circ}\right)$ for the second monitoring experiment. The M4 intensity increased by 50\%. Figures S5-S7 in the Supplementary information show the $\sim 2 \%$ hopping amplitudes, which were twice than that in Figure 4 . Two high-speed Sisyphus falls occurring over five minutes appeared after three growing stepwise increments over a period of $\sim 18$ days, which were similar to the first three hopping episodes in the first 40 days in Figure 4. The inversed source-sample configuration drove the mode hopping backwards. A PT symmetry is retained by combining the space inversion $\mathrm{P}$ and the time inversion $\mathrm{T}$ revealing a more fundamental origin of the tick-tack $\Xi$ driving the mode hopping rather than the nuclear decays. Three forward steps (see Figures S6 and S7) to the Sisyphus fall in 18 days revealed the four entangled $\gamma \mathrm{s}$ at $662 \mathrm{keV}$ reported in this work. Lattice constants divided by the pairing wavelengths of $\gamma_{11} \gamma_{12}$ and $\gamma_{21} \gamma_{22}$ remained integers at the plateaus of each step, to preserve the M4 symmetry at the lattice sites.

The effective half-life of ${ }^{182} \mathrm{Ta} \gamma \mathrm{s}$ was $118.3 \pm 0.3 \mathrm{~d}$ during the first monitoring experiment, which was longer than the normal half-life of 114.13 d. Figure S5 in the Supplementary Information reveals an accelerated ${ }^{182} \mathrm{Ta}$ decay, counts of which were more than the baseline, rather than less than the baseline in Figure 5. The effective half-life was calibrated to be $109.2 \pm 0.7 \mathrm{~d}$ during the second monitoring experiment. Neither the growth in Figure S4 nor the slowdown in Figure 5 were artifacts, because in-between calibrations of the ${ }^{182} \mathrm{Ta}$ were obtained by removing the strong ${ }^{137} \mathrm{C}$ s source.

\section{Discussion}

We summarize several phenomena of the ${ }^{182} \mathrm{Ta}$ decay regarding the presence of impinging M4 $\gamma \mathrm{s}$ at $662 \mathrm{keV}$.

(1) The ${ }^{182} \mathrm{Ta} \gamma \mathrm{s}$ gave a fast negative response to the mode hopping of the $\mathrm{M} 4 \gamma$. The matter-wave grating at $662 \mathrm{keV}$ enabled the rejection of the coexisting superradiant ${ }^{182} \mathrm{Ta} \gamma \mathrm{s}$.

(2) The M4 superradiance contracted into a shallow region beneath the sample surface depending on the $\gamma$ intensity. However, the spatial shrinkage of the M4 superradiance gave more room for the ${ }^{182}$ Ta superradiance.

(3) It is clear that a Raman pumps ${ }^{182} \mathrm{Ta}$ to the allowed $\beta$ transitions, which accelerates the decay. For example, a hyper-Raman pumped ${ }^{94} \mathrm{Nb}$ to a virtual state of $\mathrm{J}^{\pi}=6^{+}, \beta$ transitions from which to two ${ }^{94}$ Mo excitations of $\mathrm{J}^{\pi}=6^{+}$were allowed (see Figure S3C in the Supplementary Information). In contrast, the slowdown ${ }^{182} \mathrm{Ta}$ decay is subtle. It is well known that a suppression effect incorporates a destructive interference between two paths, as demonstrated by the Borrmann effect [13]. Observations of the ${ }^{182} \mathrm{Ta}$ lifetimes depending on the M4 intensity has shed some light 
on the problem. The remaining questions are to construct a model of the $\beta$ decay encountering multiple branching, splittings of which are opened by the highly-concentrated mixing of M4 and E2 fields.

(4) The impinging $\mathrm{M} 4 \gamma$ activated the ${ }^{93 \mathrm{~m}} \mathrm{Nb}$ excitation, as shown by Figure S3A in the Supplementary Information. Increasing NSDW density provides a stronger matter-wave grating to promote the collective forward scattering of ${ }^{182} \mathrm{Ta} \gamma \mathrm{s}$.

(5) Superradiance of both the impinging $\gamma \mathrm{s}$ and the internal ${ }^{182} \mathrm{Ta} \gamma \mathrm{s}$ showed systematic oscillations, which were most likely driven by external sources.

The NSDW ME symmetry reveals a pseudo-scalar axion field of arbitrary winding numbers [7,27], the underlying physics of which may have been unveiled by the recent progress on the Weyl superfluid [17]. The axion electrodynamics gives rise to the electric and the "magnetic" charges on the facets, the edges, and the vertices, where the axion gradient appears $[27,28]$. It is worthwhile investigating the boojum texture with the axionic superradiance together in the near future. 1-D superradiant tubes were concentrated at the eight long edges of the sample sheet, i.e., four 3-cm edges plus four 1.5-cm edges, which allowed the reconstruction of the mutual coherence of impinging $\gamma \mathrm{s}$ to provide the near-field diffraction, as well as to recover the fringe visibility [22] from a mm-sized source. The Arago spot revealed a $0.3 \mu \mathrm{m}$ aperture, which was probably none other than the compressed diameter of the superradiant tubes at the edges.

\section{Materials and Methods}

The preparation of active samples and the applied HPGe detector have been detailed in the references [9], while the setup of the superradiant measurements is shown in Figure 1. An $\mathrm{Nb}$ sample of $99.99 \%$ purity is described in this report, which has a dimension of $1 \mathrm{~mm} \times 1.5 \mathrm{~cm} \times 3 \mathrm{~cm}$. We applied four kinds of radioactive sources in the measurements, i.e., one $2.5-\mathrm{mCi}{ }^{137} \mathrm{Cs}$, two $10-\mu \mathrm{Ci}$ ${ }^{137} \mathrm{Cs}$, two $1-\mu \mathrm{Ci}{ }^{60} \mathrm{Co}$, and one $2.3-\mu \mathrm{Ci}{ }^{152} \mathrm{Eu}$. The $2.5-\mathrm{mCi}{ }^{137} \mathrm{Cs}$ was a $3-\mathrm{mm}$ point source encapsulated in a cylinder $8 \mathrm{~mm}$ long and $6 \mathrm{~mm}$ in diameter. Two $10-\mu \mathrm{Ci}{ }^{137} \mathrm{Cs}$ were thin disks $10 \mathrm{~mm}$ in diameter and $1 \mathrm{~mm}$ thick. Two $1-\mu \mathrm{Ci}{ }^{60} \mathrm{Co}$ and the $30-\mu \mathrm{Ci}{ }^{152} \mathrm{Eu}$ were also thin disks $3 \mathrm{~mm}$ in diameter and $1 \mathrm{~mm}$ thick. The applied HPGe detector CANBERRA GC0518 (coaxial-Ge type) with $\varphi 4.25 \mathrm{~cm}$ and a length of $4.1 \mathrm{~cm}$ was located in a stabilized box with $3-\mathrm{cm} \mathrm{Pb}$ shielding. A calibration using the $10-\mu \mathrm{Ci}$ ${ }^{137}$ Cs source yielded $110 \mathrm{cps}$ with $2.5 \mathrm{~cm}$ detector-source spacing.

Two long-term monitoring experiments of superradiance were undertaken, beginning on the 1 August 2016 (1st day 0) and on 16 November 2016 (2nd day 0). We moved the ${ }^{137}$ Cs source from $\left(0.3 \mathrm{~cm}, 90^{\circ}\right)$ to $\left(0.3 \mathrm{~cm}, 270^{\circ}\right)$ in the second monitoring experiment. Two air conditioners maintained the environmental temperature at $27 \pm 0.5^{\circ} \mathrm{C}$. The air conditioners were turned off at 3 pm on 11 October 2016. They were switched on and off twice during the following week and kept off after 18 October 2016. Afterwards, the temperature varied naturally between $18^{\circ} \mathrm{C}$ and $30^{\circ} \mathrm{C}$. The long-term monitoring of the M4 superradiance was stopped and restarted several times. We calibrated the computer clock each day; it was faster than normal by $\sim 1.5 \mathrm{~s}$ per day.

\section{Conclusions}

The NSDW of the delocalized ${ }^{93 \mathrm{~m}} \mathrm{Nb}$ state entered the BEC regime at room temperature. We observed pronounced condensate-field interactions, in particular the coherent superradiance and the suppression of atomic channels. Two M4 standing waves of 4-entangled $\gamma \mathrm{s}$ at 30 and $662 \mathrm{keV}$ coexist in the superradiant region, which construct a 1-D pm matter-wave grating. Seven Sisyphus falls occurred at the moments of forward hopping in 107 days, in addition to two other backward hopping episodes also shown.

We pay particular attention to the BEC matter-wave grating of length $\mathrm{d}$ in $\mathrm{cm}$ driven by the $\gamma \mathrm{s}$ of $\lambda_{0}$ in pm. The number of matter-wave mirrors is $n d / \lambda_{0}$, where $n$ is the effective reflective index. Assuming $n \sim 1$, the Q-factor of the Fabry-Pérot resonator is proportional to $\left(d / \lambda_{0}\right)^{2}>10^{20}[29]$, 
which is useful for detecting gravitational waves [11]. The end-fire $\gamma \mathrm{s}$ performing the mode hopping are, thus, no more than a $\gamma$-ray laser emitting from a 3 -cm resonator.

Supplementary Materials: The following are available online at www.mdpi.com/2073-4352/7/7/196/s1. Evaluation of the fluctuations in Figure 5; Figure S1: Raw data of the first 107-day monitoring experiment; Figure S2: $\gamma$ counts of the first monitoring experiment in Figure S1; Figure S3: Spectra of Figure S1, Figure S5, and calibration; Figure S4: ${ }^{182}$ Ta $\gamma$ count rates; Figure S5: Raw data of the second 40-day monitoring experiment; Figure S6: Data in Figure S5 incorporating the hourly smoothing (61 data); Figure S7: $\gamma$ counts of the second monitoring experiment.

Acknowledgments: We wish to thank Ian Hsu for the discussion of the $\gamma$-ray nanoimaging; Ite A. Yu for the discussion on BEC, mode hopping, radiation trapping, and electromagnetic-induced transparency; Shiang-Huei Jiang for the strong ${ }^{137} \mathrm{Cs}$ source; Yaw-Wen Yang and Keng S. Liang for discussion of the catalysis; Tai-Ching Fan for the evaluation of ${ }^{94} \mathrm{Nb}$ and the energy calibration of the HPGe detector; Wei-Che Hsieh for the long-term monitoring code; Ben-Li Young for setting up the computer software; and Kuang-You Chen for the evaluation of the ${ }^{182} \mathrm{Ta}$ decay. Ting-Han Lin, Shun-Chi Wu, and Chih-Hao Lee thank the Ministry of Science and Technology of Taiwan, R.O.C., for supporting this work under contract nos. MOST 105-2221-E-007-090 and MOST-105-2112-M-007-023.

Author Contributions: Yao Cheng undertook the theoretical and the experimental work; Tin-Han Lin and Shun-Chi Wu undertook the data preparation; and Chih-Hao Lee was responsible for the sample activation.

Conflicts of Interest: The corresponding authors declare no competing financial interests and the work has been undertaken without any financial support from the Chinese NSFC, except the sample donation by the Ho Kang technology Co.

\section{Abbreviations}

The following abbreviations are used in the manuscript:

$\begin{array}{ll}\text { BEC } & \text { Bose-Einstein Condensation } \\ \text { ODLRO } & \text { off-diagonal long-ranged order } \\ \text { ME } & \text { magnetoelectric effect } \\ \text { NSDW } & \text { nuclear spin-density wave } \\ \text { SAC } & \text { suppression of atomic channels } \\ \text { BBL } & \text { broad-band x-rays in low-energy range } \\ \text { BBM } & \text { broad-band x-rays in middle-energy range } \\ \text { BBH } & \text { broad-band x-rays in high-energy range } \\ \text { HPGe } & \text { high-purity germanium } \\ \text { Cps } & \text { counts per second }\end{array}$

\section{References}

1. Anderson, M.H.; Ensher, J.R.; Matthews, M.R.; Wieman, C.E.; Cornell, E.A. Observation of Bose-Einstein condensation in a dilute atomic Vapor. Science 1995, 269, 198-201. [CrossRef] [PubMed]

2. Davis, K.B.; Mewes, M.-O.; Andrews, M.R.; van Druten, N.J.; Durfee, D.S.; Kurn, D.M.; Ketterle, W. Bose-Einstein condensation in a gas of sodium atoms. Phys. Rev. Lett. 1995, 75, 3969-3973. [CrossRef] [PubMed]

3. Yang, C.N. Concept of off-diagonal long-range order and the quantum phases of liquid He and of superconductors. Rev. Mod. Phys. 1964, 34, 694-703. [CrossRef]

4. Ueda, M. Fundamentals of Bose-Einstein Condensation. In Fundamentals and New Frontiers of Bose-Einstein Condensation; Ryan, B., Ed.; World Scientific Co., Pte. Ltd.: Singapore, 2010; pp. 1-32.

5. Deng, H.; Haung, H.; Yamamoto, Y. Exciton-polariton Bose-Einstein condensation. Rev. Mod. Phys. 2010, 82, 1489-1537. [CrossRef]

6. Carusotto, I.; Ciuti, C. Quantum fluid of light. Rev. Mod. Phys. 2013, 85, 299-366. [CrossRef]

7. Cheng, Y.; Guo, Z.-Y.; Liu, Y.-L.; Lee, C.-H.; Young, B.-L. Magnetoelectric effect induced by the delocalised $93 \mathrm{~m} \mathrm{Nb}$ state. Radiat. Eff. Defects Solids 2015, 170, 43-54. [CrossRef]

8. Liu, Y.-Y.; Cheng, Y. Impurity channels of the long-lived Mossbauer effect. Sci. Rep. 2015, 5, 15741. [CrossRef] [PubMed] 
9. Cheng, Y.; Yang, S.-H.; Lan, M.; Lee, C.-H. Observations on the long-lived Mossbauer effects of ${ }^{93 \mathrm{~m}} \mathrm{Nb}$. Sci. Rep. 2016, 6, 36144. [CrossRef] [PubMed]

10. Cheng, Y.; Xia, B.; Chen, C.P. Cooling effect in emissions of ${ }^{103 \mathrm{~m}} \mathrm{Rh}$ excited by bremsstrahlung. Phys. Scr. 2009, 79, 055703. [CrossRef]

11. Cheng, Y. A possible scheme for measuring gravitational waves using a spinful quantum fluid. In Proceedings of the ISEG5-Fifth International Symposium on Experimental Gravitation (EPJ Web of Conferences 2014), Nanjing, China, 8-13 July 2013; p. 02001.

12. Maggiore, M. Spontaneous symmetry breaking. In A Modern Introduction to Quantum Field Theory; Oxford University Press: New York, NY, USA, 2007; pp. 253-265.

13. Hannon, J.P.; Trammell, G.T. Coherent $\gamma$-ray optics. Hyperfine Interact. 1999, 123, 127-274. [CrossRef]

14. Fox, M. Resonant light-atom interactions. In Quantum Optics; Oxford University Press: New York, NY, USA, 2006; pp. 167-193.

15. Greiner, M.; Mandel, O.; Hansch, T.W.; Bloch, I. Collapse and revival of the matter wave field of a Bose-Einstein condensate. Nature 2002, 419, 51-54. [CrossRef] [PubMed]

16. Khaymovich, I.M.; Silaev, M.A. Magnetic resonance within vortex cores in the B phase of superfluid ${ }^{3} \mathrm{He}$. Phys. Rev. B 2010, 82, 094507. [CrossRef]

17. Mizushima, T.; Tsutsumi, Y.; Kawakami, T.; Sato, M.; Ichioka, M.; Machida, K. Symmetry-Protected Topological Superfluids and Superconductors-From the Basics to ${ }^{3} \mathrm{He}$ - J. Phys. Soc. Jpn. 2016, 85, 022001. [CrossRef]

18. Qi, X.-L.; Zhang, S.C. Topological insulators and superconductors. Rev. Mod. Phys. 2011, 83, $1057-1101$. [CrossRef]

19. Inouye, S.; Chikkatur, A.P.; Stamper-Kurn, D.M.; Stenger, J.; Pritchard, D.E.; Ketterle, W. Superradiant Rayleigh scattering from a Bose-Einstein condensate. Science 1999, 285, 571-574. [CrossRef] [PubMed]

20. Yoshikawa, Y.; Torii, Y.; Kuga, T. Superradiant light scattering from thermal atomic vapors. Phys. Rev. Lett. 2005, 94, 83602-83605. [CrossRef] [PubMed]

21. Ritsch, H.; Domokos, P.; Brennecke, F.; Esslinger, T. Cold atoms in cavity-generated dynamical optical potentials. Rev. Mod. Phys. 2013, 85, 553-601. [CrossRef]

22. Hecht, E. Optics, 4th ed.; Addison Wesley: San Francisco, CA, USA, 2002.

23. Krause, M.O.; Oliver, J.H. Natural widths of atomic K and L levels, K $\alpha$ X-ray lines and several KLL Auger lines. J. Phys. Chem. Ref. Data 1979, 8, 329-338. [CrossRef]

24. Belger, M.; Schimming, R.; Wunsch, V. A survey on Huygens' principle. Z. Anal. Anwend. 1997, 16, 9-36. [CrossRef]

25. Baumann, K.; Guerlin, C.; Brennecke, F.; Esslinger, T. Dicke quantum phase transition with a superfluid gas in an optic cavity. Nature 2010, 464, 1301-1306. [CrossRef] [PubMed]

26. Gupta, S.; Moore, K.L.; Murch, K.W.; Stamper-Kurn, D.M. Cavity Nonlinear Optics at Low Photon Numbers from Collective Atomic Motion. Phys. Rev. Lett. 2007, 99, 213601. [CrossRef] [PubMed]

27. Wilczek, F. Two applications of axion electrodynamics. Phys. Rev. Lett. 1987, 58, 1799-1802. [CrossRef] [PubMed]

28. Lee, Y.L.; Park, H.C.; Ihm, J.; Son, Y.-W. Manifestation of axion electrodynamics through magnetic ordering on edges of a topological insulator. Proc. Natl. Acad. Sci. USA 2015, 112, 11514-11518. [CrossRef] [PubMed]

29. Braginsky, V.B.; Vyatchanin, S.P. Low quantum noise tranquilizer for Fabry-Perot interferometer. Phys. Lett. A 2002, 293, 228-234. [CrossRef]

(C) 2017 by the authors. Licensee MDPI, Basel, Switzerland. This article is an open access article distributed under the terms and conditions of the Creative Commons Attribution (CC BY) license (http://creativecommons.org/licenses/by/4.0/). 